\title{
Huge light trapping with surface scattering structures in a flexible transparent substrate
}

\author{
R. Barille ${ }^{1}$, F. Cousseau ${ }^{1}$, M. Loumaigne ${ }^{1}$, S. Zielinska ${ }^{2}$, E. Ortyl ${ }^{2}$, S. Gholaghasemi ${ }^{1}$ \\ ${ }^{I}$ Moltech-Anjou, 2 Boulevard Lavoisier, 49045, Angers, France \\ ${ }^{2}$ Wroclaw University of Technology, Department of Polymer Engineering and Technology, \\ 50-370 Wroclaw, Poland. \\ regis.barille@univ-angers.fr
}

\begin{abstract}
We show in this study the fabrication of quasi-random and Gaussian random structures (2D random structures) with a photo-induced nanostructured material for the control of lightmatter interactions. This material offers a novel approach for the design of richer Fourier spectra by using a pattern with different periodic structures and sub-structures that allow the control of the Fourier components at will. These structures can be used to achieve a huge efficient omnidirectional light coupling in thin films and energy harvesting for solar cells.

OCIS codes: $160.4236,160.4760,220.4241,230.1950$
\end{abstract}

\section{Introduction}

Disordered photonics has experienced a burst of activity in the last two decades [1,2]. The fabrication of nanostructured materials for the control of light-matter interactions becomes particularly important for the improvement of different phenomena involving a highly scattering of light. Different techniques have been explored as photonic crystals, self-assembly of particles, or randomly distributed voids created by a femtosecond laser interaction, etc. However a strategy proposing an easy fabrication method, a good reproducibility, a wide area fabrication and cheap materials would be appreciated. In this goal we propose in this study the use of azopolymer for the fabrication of 2D quasi random structures for light trapping. The technology is based on azopolymer. Azobenzene derivatives are one of the most frequently studied classes of switchable compounds. The azobenzene functionality exhibits cis-trans isomerization. The thermodynamically stable trans isomer can be converted to the cis isomer by a light stimuli whose wavelengths is in the absorption band. Engineering azopolymer materials can lead to self-induced patterns by photoisomerization induced by light on the surface of thin films. One example of such induced patterns is the production of self-organized structures leading to gratings on the surface of side-chain azobenzene graphted copolymer [3]. We study and compare two types of random surface for light trapping.

\section{Results}
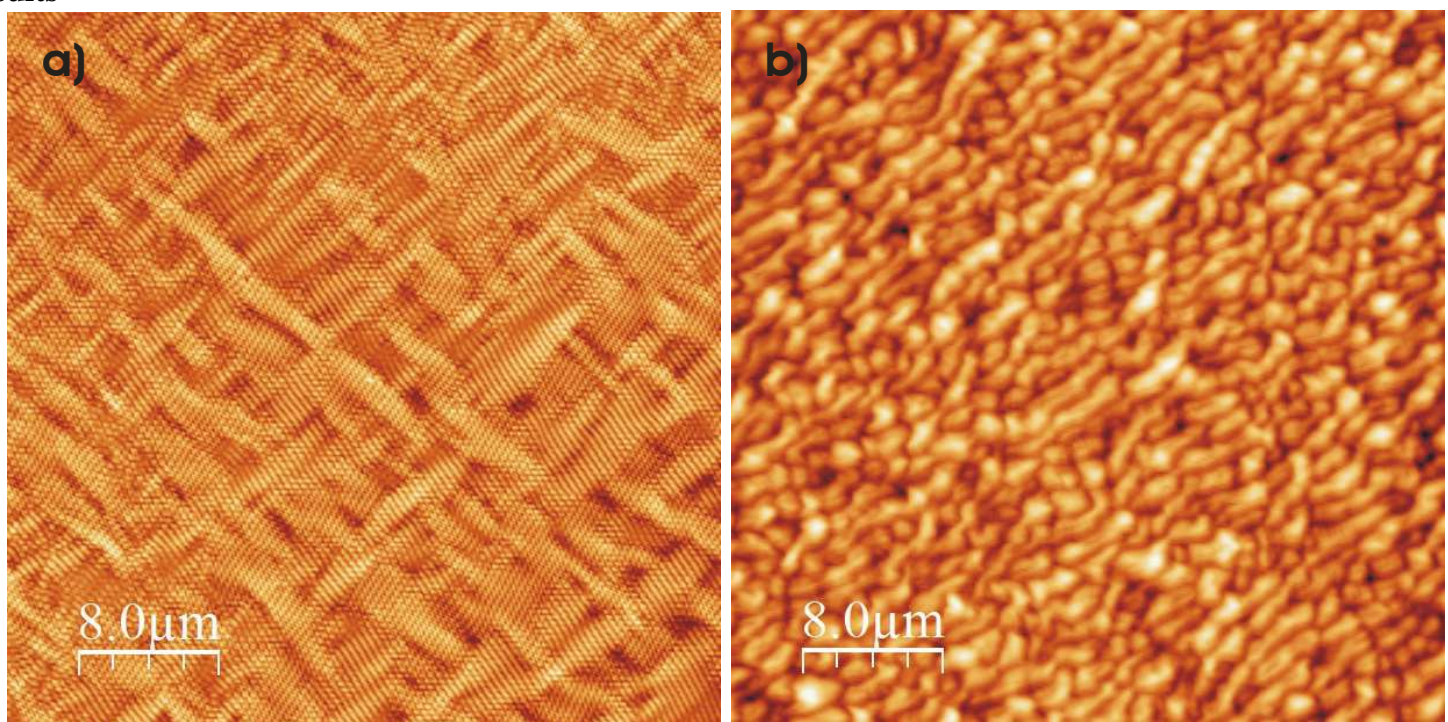

Fig. 1: AFM measurement of the surface pattern obtained after illumination. of a chiral azopolymer thin film. a) Surface pattern of a quasi random grating surface, b) random surface pattern 
The azopolymer consists in the polymerization of three-component mixtures of azobenzene group containing N-[4-[(E)-[4-[bis(2-hydroxyethyl) amino] phenyl] azo]phenyl]sulfonyl benzamide, chiral 2,3O-benzylidene-D-threitol and isophorone-diisocyanate (molar ratio of azobenzene derivative, chiraldiol and diisocyanatein that mixture was 1:1:2). The copolymers showed reversible photochromic properties under illumination with light by change of UV-vis spectra and ellipsometric parameters. The chiro-optical properties of the materials were investigated by circular dichroism spectroscopy.

The experimental setup consists in a laser beam from a diode pump solid state laser at $\lambda=473 \mathrm{~nm}$ with a power of $50 \mathrm{~mW}$. The laser is used to excite the azopolymer absorption close to its absorption maximum. The size of the collimated laser beam impinging on the polymer sample is adjusted with a Kepler-type afocal system and is a plane wave. The sample is irradiated with a defect beam size of $2 \mathrm{~mm}$ diameter at $1 / \mathrm{e}^{2}$. The power density is $0.7 \mathrm{~W} / \mathrm{cm}^{2}$. A combination of lens is used to measure the first order diffraction intensity and allows us evaluating the photoinduced structuration on the surface of the sample and the molecular rearrangement leading to a mass transport.

The figure 1 shows the surface gratings obtained after illumination by the laser beam. The topographic pattern was measured with an AFM. We note on figure 1a) multiple gratings inscribed randomly on the surface with two different directions. The pitch of the gratings figure 1a) is about $0.9 \mu \mathrm{m}$ and the angle between the two gratings is about $45^{\circ}$. The topography of the figure $1 \mathrm{~b}$ ) is a quasi-random pattern nearly a perfect Gaussian surface. The cell size has an average perimeter in the order of $2.3 \mu \mathrm{m}$. The figure $2 \mathrm{a}$ ) shows that the quasi random grating pattern allows the light to be spatially diffracted in an omni-directional way.

a)

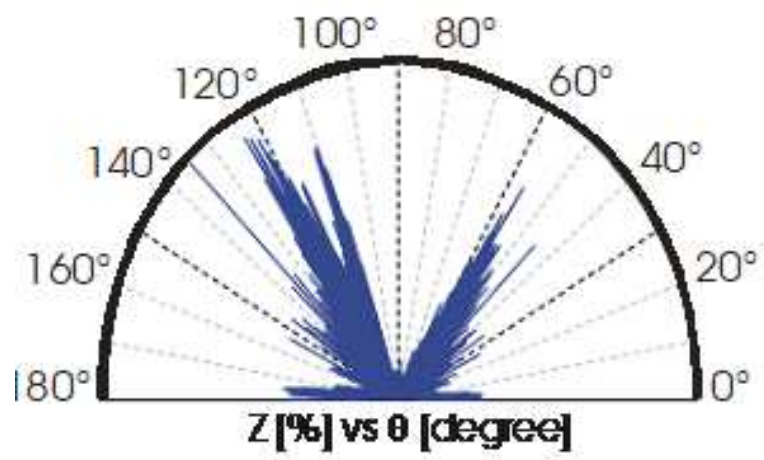

b)

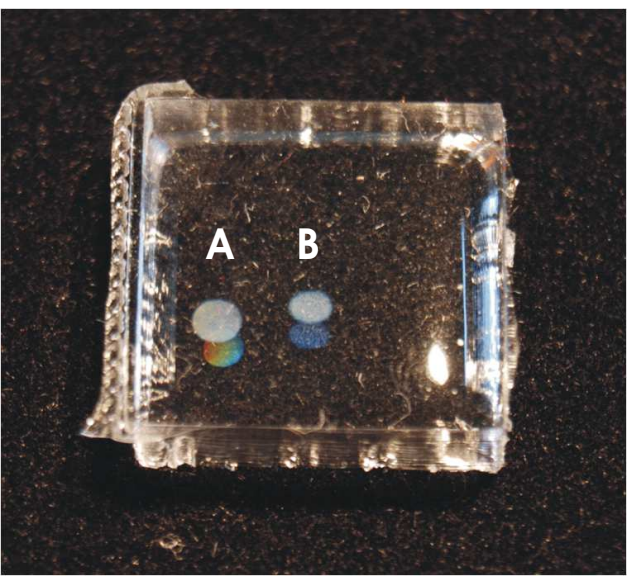

c)

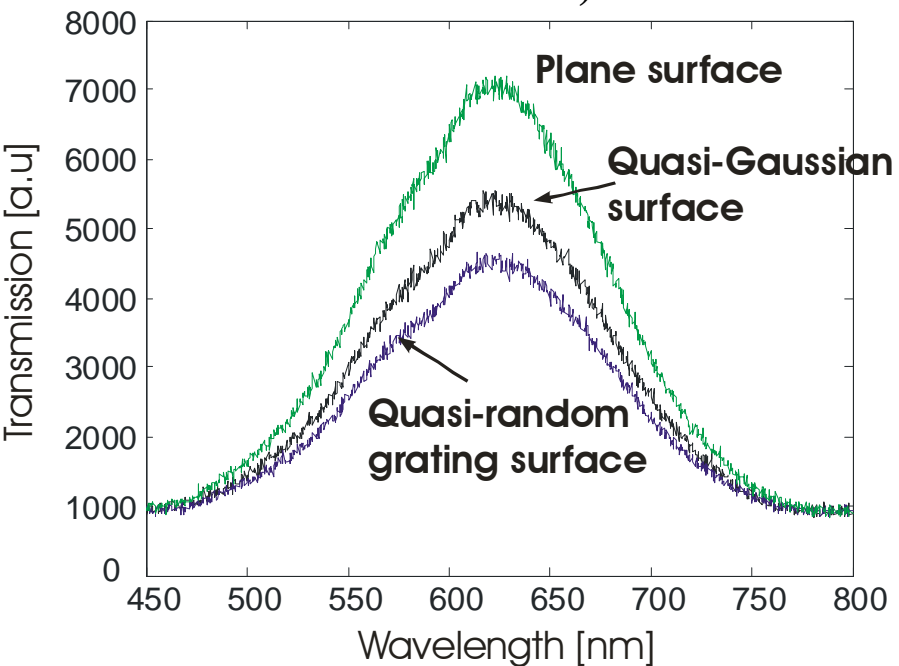

Fig.10. a) directionality of the light emission from the surface b) Replica modeling of the surface pattern of the random grating and quasi random grating on an elastomeric material A and B respectively. b) Transmission spectrum of the elastomeric surface with quasi-random gratings, a Gaussian surface and comparison with the reference plane surface. 
We replicated the photoinduced surface of the two photoinduced surfaces on a widely used elastomeric material, (poly(dimethylsiloxane (PDMS)). The PDMS sample is largely transparent with a transmission above $95 \%$ in the visible region of the spectrum. Taking the plane PDMS surface as a reference, we measured the light extinction, induced by the patterned surface, for both materials. We found an increase of the light extinction ratio of $28 \%$ and $41 \%$ for the quasi Gaussian surface and the quasi-random grating surface, respectively.

In conclusion we have presented for the first time a cheap method for the fabrication of 2D random structures able to couple light into a thin film with an omni-direction and a record in the light trapping [4]. This organic material can be suitable for energy harvesting of organic solar cells or OLEDs.

\section{References}

[1] J. Binghi, V. M. Murukeshan, 'Speckle lithography for fabricating Gaussian, quasi random 2D structures and black silicon structures', Sci. Rep., doi:10.1038/srep18452.

[2] E. R. Martins, J. Li, Y. Liu, V. Despaw, A. Chen, J. Zhou, T. F. Krauss, 'Deterministic quasi-random nanostructures for phton control', Nat. Comm., doi:10.1038/ncomms 3665

[3] R. Barillé, P. Tajalli, S. Kucharski, E. Ortyl, J.M. Nunzi, "Photoinduced deformation of azopolymernanometric spheres", Appl. Phys. Lett. 96, $163104(2010)$

[4] Sh. Golghasemi Sorkhabi, S. Ahmadi-Kandjani, F. Cousseau, M. Loumaigne, S. Zielinska, E. Ortyl, and R. Barille, 'Surface quasi periodic and random structures based on nanomotor lithography for light trapping', Journal of Applied Physics 122, 015303 (2017) 